Original Article

\title{
Effects of wearing functional foot orthotic on pelvic angle among college students in their 20s with flatfoot
}

\author{
KWAngYong Park, MS ${ }^{1)}$ \\ 1) Department of Rehabilitation Technology, Korea Nazarene University: 456 Sangyong-dong, \\ Seobuk-gu, Cheonan, Chungnam 331-718, Republic of Korea
}

\begin{abstract}
Purpose] This study examined the impact of wearing a functional foot orthotic on the pelvic angle of young adults who have flatfoot. [Subjects and Methods] A total of 15 college students diagnosed with flatfoot were included in the study. Changes in the pelvic angle in the stance period during walking before and after orthotic use were measured using a VICON Motion System (VICON, Hansung, Korea). The data were analyzed using SPSS 12.0 for Windows. [Results] In the experimental group, the pelvic angle during the mid-stance and mid-swing periods of the gait cycle significantly decreased on the left and right sides after wearing the orthotic, compared to the measurements taken before orthotic usage. The pelvic angle change on the left and right sides also decreased after wearing the orthotic, though this difference was not significant. [Conclusion] The pelvic angle of college students with flatfoot decreased after they wore orthotics. This implies that wearing orthotic shoes can greatly benefit individuals during normal walking by promoting passive changes that decrease the pelvic angle.

Key words: Flat-foot, VICON, Gait cycle
\end{abstract}

(This article was submitted Oct. 13, 2016, and was accepted Nov. 24, 2016)

\section{INTRODUCTION}

Walking is one of the most fundamental actions required for human life, and feet play a particularly important role in supporting the human body and maintaining balance during walking ${ }^{1}$. Because of this, incorrect walking habits primarily appear as an imbalance between the two feet. In particular, flatfoot is characterized by a medial longitudinal arch that is lowered in a chronic or abnormal way, resulting in hyperextension of the plantar fascia, spring ligament, and tendon of the tibialis posterior ${ }^{2}$. As the ability to receive and disperse weight decreases due to the abnormality of the sole structure, excessive compensation by the outer muscles occurs and causes foot imbalance ${ }^{3)}$. Hence, flatfoot walking induces excessive pronation of the feet, which delivers the weight load to the tibia, causing pain in the tibia and knee. This can result in damage to the lower limb4).

In a study using functional foot orthotics (FFOs) to solve the problem of foot deformity, Pratt $^{5)}$ examined the prevention or correction of deformity, formation of sufficient supporting ground, promotion of standing or walking exercise, and improvement of walking efficiency. Feet play an important role in the lower kinetic chain; they distribute the weight load generated during exercise and disperse it in the stance period during walking. Song ${ }^{6}$ mentioned that orthotics decrease both pronation and internal rotation of the tibia. Moreover, an appropriate shoe liner and orthotic can decrease muscle activity and provide comfort, while increasing exercise performance ability ${ }^{7,8)}$. As the FFO supports a balanced weight distribution in the plantar area and arch, it aids in efficient shock absorption, including the ground reaction generated during walking or running, which reduces pain and unstable joint motion ${ }^{9}$.

As such, diverse studies have been conducted examining walking function and orthotic use. Although there are a number

Corresponding author. KwangYong Park (E-mail: bracep@kornu.ac.kr)

(C2017 The Society of Physical Therapy Science. Published by IPEC Inc.

This is an open-access article distributed under the terms of the Creative Commons Attribution Non-Commercial No Derivatives (by-nc-nd) License $<$ http://creativecommons.org/licenses/by-nc-nd/4.0/>. 
Table 1. General participant characteristics

\begin{tabular}{lc}
\hline & Subjects $(\mathrm{n}=15)$ \\
\hline Gender $(\mathrm{M} / \mathrm{F})$ & $8 / 7$ \\
Age $(\mathrm{yrs})$ & $21.1 \pm 3.4$ \\
Height $(\mathrm{cm})$ & $162.1 \pm 6.1$ \\
Weight $(\mathrm{kg})$ & $63.1 \pm 7.1$ \\
Calcaneal pitch angle $\left(^{\circ}\right)$ & $13.5 \pm 1.2$ \\
\hline
\end{tabular}

Values are presented as the mean $\pm \mathrm{SD}$

of studies analyzing the ankle and knee, research studying the pelvis, which is used as a center point of the body, during walking is scarce. Hence, this study examined the impact of wearing FFOs on the pelvic angle among young adults who have flatfoot, using a VICON Motion System device.

\section{SUBJECTS AND METHODS}

The subjects of this study consisted of 15 college students enrolled in University K, located in Cheonan City, Korea, from September 1 to 20 in 2015. The study included participants with no musculoskeletal disease who were diagnosed with flatfoot and have a calcaneal pitch angle less than $15^{\circ}$, indicated by radiological measurements. The selected subjects fully understood the study and provided written, informed consent. This study acquired approval from the Clinical Test Screening Committee at Korea Nazarene University and was reviewed according to the Declaration of Helsinki. The general characteristics of the research subjects who participated in this study are summarized in Table 1.

The FFOs worn by the subjects in this study were customized for each individual's foot shape and created with thermoplastic materials. A high-density resistance elastic pad, cup sole for the plantar arch, low-elasticity pad for shock absorption in the heel, and ethylenevinylacetate (EVA) were used to create the FFOs. When designing the foot orthotic for a subject, the researcher positioned the heel bone vertical to the ground and let the subtalar joint maintain its natural position. While preventing pronation of the subject's feet and excluding excessive foot movement, measurements were taken and an evaluation was performed while weight was loaded under the realigned condition of the foot. Next, the orthotic was cast and Alfoots Inc. in Korea was commissioned for its fabrication. Specifically, the experimenter measured and evaluated the subjects' feet and molded both foot shapes using Pedilen foam. With a prepared positive plaster model, plaster correction was performed (checking pressure points or sensitive parts of the feet), followed by shell production and modeling with thermoplastic. Next, posting and grinding (trimming and alignment adjustment) were performed and the production of the foot orthotic was finalized with a covering material ${ }^{10,11)}$

The study equipment consisted of six MX-F40 cameras (VICON, Hansung, Korea), two OR6-7 force plates (AMTI, USA), and Nexus software. Each MX camera forms the two-dimensional images obtained from optical markers into threedimensional images, so that in addition to the location data of the markers, measurements for each segment of the body can be obtained. It is capable of measurement up to 2,000 fps. The force plates express the ground reaction force of both feet during walking as vectors. Moreover, an Ultranet system synchronized the motor mechanic data from the camera and the kinematic data from the force plate into the same frame ${ }^{12,13)}$.

To compute the pelvic joint angle, a Plug-in Gait model, based on the Newington-Helen Hayes gait model, was used. This was measured by the three-dimensional motion capture device from the VICON Motion System. The local coordinate system of each segment was created using the three-dimensional spatial coordinates of each measured marker point, and a Euler algorithm was derived. Each gait cycle was differentiated using the heel strike as a standard, and one gait cycle of walking from each subject was extracted for time normalization ${ }^{12)}$. In this experiment, only the results from the mid-swing and mid-stance periods of the gait cycle were extracted, which provides the highest and lowest points, respectively.

In this study, to measure the change in the pelvic angle, walking on a previously fabricated Walkway before and after wearing the customized FFOs was analyzed using high-quality 3D cameras and the VICON Motion System (Vicon, Oxford, UK). To measure the pelvic angle in the stance period during walking, markers were placed on the anterior superior iliac spine (ASIS) and anterior inferior iliac spine (AIIS) bilaterally. Moreover, the dynamic change was quantified to compute the difference in the pelvic angle on the left and right sides. All subjects performed a preliminary motion five times to become familiar with the test. Data for three walking trials were processed for quantitative computation and their average values were analyzed. Data analysis was conducted using SPSS 12.0 for Windows. The changes before and after wearing the orthotic were analyzed using paired t-tests. The statistical significance level was set at $\mathrm{p}<0.05$. 
Table 2. Comparison of the pelvic angle before and after wearing the foot orthotic

\begin{tabular}{|c|c|c|c|c|c|c|c|c|}
\hline & \multicolumn{2}{|c|}{ Pre-stance $\left(^{\circ}\right)$} & \multicolumn{2}{|c|}{ Mid-stance $\left({ }^{\circ}\right)$} & \multicolumn{2}{|c|}{ Pre-swing $\left(^{\circ}\right)$} & \multicolumn{2}{|c|}{ Mid-swing $\left(^{\circ}\right)$} \\
\hline & pretest & posttest & pretest & posttest & pretest & pretest & pretest & pretest \\
\hline Right & $-4.2 \pm 0.2$ & $-2.6 \pm 0.6^{*}$ & $-6.5 \pm 0.6$ & $-4.4 \pm 0.2^{*}$ & $3.5 \pm 0.2$ & $1.9 \pm 0.5^{*}$ & $7.5 \pm 0.5$ & $4.8 \pm 0.2^{*}$ \\
\hline Left & $-4.4 \pm 0.7$ & $-2.2 \pm 0.3^{*}$ & $-6.2 \pm 0.5$ & $-4.2 \pm 0.4^{*}$ & $3.0 \pm 0.3$ & $2.1 \pm 0.7^{*}$ & $7.0 \pm 0.7$ & $5.0 \pm 0.4^{*}$ \\
\hline
\end{tabular}

Values are presented as the mean $\pm \mathrm{SE}$

*Significant difference from the pre-test value, $\mathrm{p}<0.05$

\section{RESULTS}

Examining the change in the pelvic angle in the experimental group before and after wearing the FFO revealed large changes in the pelvic angle on both the left and right sides during the pre-stance and mid-stance and pre-swing and midswing periods of the gait cycle before wearing the orthotic. These changes decreased significantly after wearing the orthotic $(p<0.05)$. However, no significant differences were observed in the pelvic angle changes of the left versus right side after wearing the orthotic $(\mathrm{p}>0.05$; Table 2$)$.

\section{DISCUSSION}

This study examined the change in the pelvic angle at the mid-stance and mid-swing phases of walking before and after wearing FFOs, in a sample of young adults who have flatfoot, with the expectation that wearing FFOs will affect the pelvic angle during the gait cycle among those with flatfoot. Examining the change in the pelvic angle during the stance period before and after wearing the FFO revealed that the angle significantly decreased on the left and right sides in the pre-stance and mid-stance and pre-swing and mid-swing periods after wearing the FFO. Although the angle decreased after wearing the FFO, compared to before, the decrease was not significant. This implies that wearing a custom-fit FFO ameliorates tension in the sole muscle and fascia and prevents the decrease of the plantar arch during the mid-stance and mid-swing periods in flatfoot walking, resulting in a decrease in the pelvic angle.

An FFO can reduce the height change of the foot arch, which eventually prevents its collapse during dynamic movement. Moreover, FFOs support effective energy consumption during walking by reducing fatigue in the foot muscles, which is frequently observed among flatfoot patients ${ }^{13)}$. They also help reverse abnormal changes in the joints and revert the joint positions back to their natural locations. Hence, a change was observed that restricted movement to the side of the pelvis joint in the sagittal plane of the pelvis after wearing FFOs. $\mathrm{Wu}^{14)}$ reported that foot orthotics are used for foot alignment and support, prevention and correction of foot deformity, and improvement of foot function. If individuals with flatfoot develop disorders of the joints and feet, FFOs can alter the walking mechanism, which consequently affects the other lower limb joints ${ }^{15}$. Moreover, they help evenly disperse shock on the sole by compensating for functional insufficiencies in the flat foot in each phase of walking ${ }^{16}$. As such, the improved results in the lower limb and foot accomplished by wearing an FFO that are reported in previous literature were likely accomplished through organic changes in the pelvic angle via the functional changes observed in this study. Future studies based on these results that examine complex organic changes in all joints of the lower limb after wearing FFOs will contribute to further advancement in orthotics.

\section{ACKNOWLEDGEMENT}

This research was supported by the Korean Nazarene University Research Grants 2017.

\section{REFERENCES}

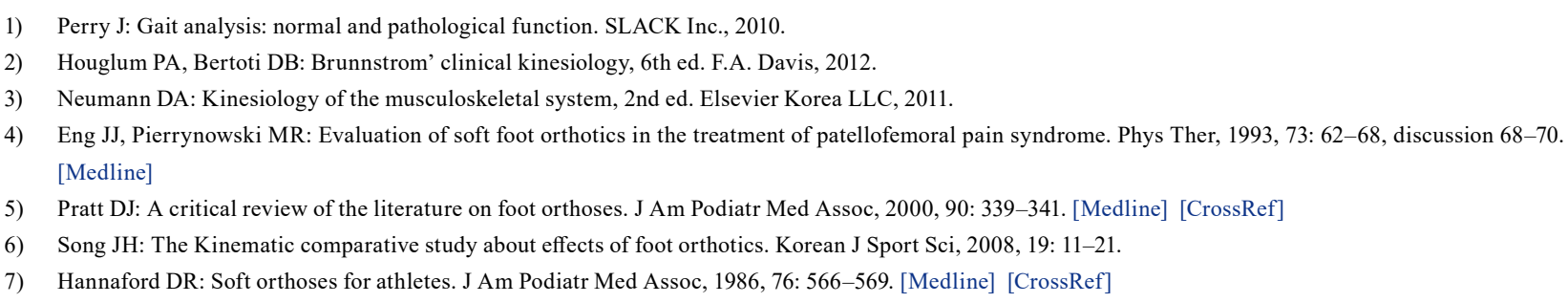


8) Nigg BM, Nurse MA, Stefanyshyn DJ: Shoe inserts and orthotics for sport and physical activities. Med Sci Sports Exerc, 1999, 31: S421-S428. [Medline] [CrossRef]

9) Gross ML, Napoli RC: Treatment of lower extremity injuries with orthotics shoe insert. Sprots Med, 1993, 15: 99-70.

10) Takata Y, Matsuoka S, Okumura N, et al.: Standing balance on the ground -the influence of flatfeet and insoles. J Phys Ther Sci, 2013, 25: 1519-1521. [Medline] [CrossRef]

11) Lee Y, Her JG, Choi Y, et al.: Effects of ankle-foot orthosis on lower limb muscle activities and static balance of stroke patients author's names. J Phys Ther Sci, 2014, 26: 179-182. [Medline] [CrossRef]

12) Han JM, Kim HA, Koo JP, et al.: Effects respiratory muscle activity in stroke patients after feedback breathing exercise. IAPTR, 2013, 4: 552-556.

13) Franco AH: Pes cavus and pes planus. Analyses and treatment. Phys Ther, 1987, 67: 688-694. [Medline]

14) Wu KK: Foot orthoses, principles and clinical applications. Baltimore: Williams and Wilkins, 1990, p 97.

15) Magee DJ: Orthopedic physical assessment, 3rd ed. Philadelphia: WB Saunders, 1997.

16) Hertel J, Sloss BR, Earl JE: Effect of foot orthotics on quadriceps and gluteus medius electromyographic activity during selected exercises. Arch Phys Med Rehabil, 2005, 86: 26-30. [Medline] [CrossRef] 